\title{
Au commencement était la dissidence
}

Création et séparation au sein du pentecôtisme ghanéen

\section{Sandra Fancello}

\section{(2) OpenEdition}

Journals

Édition électronique

URL : http://journals.openedition.org/assr/1507

DOI : $10.4000 /$ assr. 1507

ISSN : $1777-5825$

Éditeur

Éditions de l'EHESS

Édition imprimée

Date de publication : 1 avril 2003

Pagination : 45-55

ISBN : 2-222-96732-5

ISSN : 0335-5985

Référence électronique

Sandra Fancello, «Au commencement était la dissidence », Archives de sciences sociales des religions [En ligne], 122 I avril - juin 2003, mis en ligne le 10 novembre 2005, consulté le 19 avril 2019. URL : http://journals.openedition.org/assr/1507 ; DOI : 10.4000/assr.1507 


\title{
AU COMMENCEMENT ÉTAIT LA DISSIDENCE Création et séparation au sein du pentecôtisme ghanéen
}

\author{
À propos de : \\ LARBI (Emmanuel Kingsley), Pentecostalism. The \\ Eddies of Ghanaian Christianity, Accra-Ghana, CPCS \\ (Centre of Pentecostal and Charismatic Studies), 2001, \\ 517 p (avant-propos de Paul Gifford) (coll. «SAPC \\ [Studies in African Pentecostal Christianity]», \\ series 1). \\ MEYER (Birgit), Translating The Devil. Religion and \\ Modernity Among The Ewe in Ghana, Édimbourg, \\ Edinburgh University Press, 1999, 260 p. (bibliogr., \\ glossaire, cartes).
}

Dans le contexte d'émergence et de diffusion des pentecôtismes africains, le rôle des pays anglophones, de l'Afrique de l'Ouest à l'Afrique du Sud, est déterminant. Dès les premières missions méthodistes, baptistes et anglicanes, des pays comme le Ghana, le Nigeria, le Liberia et la Sierra Leone, constituent des plaques tournantes de l'expansion des protestantismes au sein de l'Afrique de l'Ouest, notamment dans sa partie francophone. La situation géographique de chacun de ces pays, limitrophes ou littéralement entourés de pays francophones, les prédisposait à une logique d'échange et d'influence réciproque. Depuis l'épopée ivoirienne et ghanéenne du Prophète libérien W. W. Harris (1913-1914) que certains considèrent comme le précurseur du mouvement pentecôtiste dans ces pays (1) et la naissance des Églises Aladura du Nigeria (1930) à partir desquelles se sont constituées nombre d'Églises prophétiques africaines au cours du XX $\mathrm{X}^{\mathrm{e}}$ siècle, la créativité religieuse de ces pays se confirme, contribuant à dessiner un paysage religieux ouest africain foisonnant et multiple. Concernant le Ghana et le Nigeria, de nombreux chercheurs anglophones se sont depuis longtemps engagés dans des études de sociologie de la conversion et d'histoire des religions protestantes. Ces pays disposent aujourd'hui d'une littérature abondante sur l'histoire des Églises africaines,

(1) David A. Shank, «Le Pentecôtisme du prophète William Wadé Harris », Archives de Sciences Sociales des Religions, $\mathrm{n}^{\circ} 105,1999$, pp. 51-70. 
contrairement à beaucoup de pays francophones ouest-africains comme le BurkinaFaso, la Côte-d'Ivoire et le Togo, exception faite du Bénin qui a fait l'objet de nombreux travaux.

Précisons d'emblée que les deux ouvrages cités, bien que centrés sur les formes de l'appropriation du pentecôtisme par les Églises ghanéennes, ne portent pas sur la même zone du Ghana et procèdent chacun d'une démarche différente. Le travail de B.M. est issu d'une thèse d'anthropologie préparée à la School for Social Science Research de l'université d'Amsterdam, en collaboration avec le Department of African Studies - Sociology de l'université de Legon (Accra). Les trois premiers chapitres portent sur l'histoire missionnaire en pays Ewé et l'attrait du christianisme perçu comme "new way of life " dans la société coloniale. Sur le plan ethnographique, l'auteur centre son analyse sur l'Evangelical Presbyterian Church (EPC), fondée à l'initiative des missionnaires piétistes allemands de la Norddeutsche Missionsgesellschaft (NMG) installés en pays Peki (Ewé (2)) depuis 1847. La rencontre religieuse, perçue comme la première étape de la «longue conversation » (3) des Ewé avec les Blancs, associée à l'investissement des missionnaires dans l'apprentissage des langues locales, se traduit par un travail de traduction et de "vernacularisation » des notions du vocabulaire chrétien dans la langue ewé. Cette «vernacularisation » va de pair avec une forme de "diabolisation » des catégories traditionnelles ewé, mobilisant les catégories du Mal et les différents aspects du Malin, comme l'évoque le titre de l'auteur, «traduire le Diable ». Cette diabolisation des esprits «païens » qui, du même coup, consacre leurs pouvoirs, place l'image du Diable au centre du discours chrétien, comme en témoigne la première impression de l'auteur : "I began to realise the immense importance of the image of the Devil in local appropriations of Christianity among the Ewe » (xvii). Ainsi, la figure du Diable crée un lien privilégié et paradoxal entre la religion chrétienne et la religion Ewé. La rencontre entre une tradition chrétienne de diabologie et la religion Ewé aboutit à un "discours chrétien Ewé » (Ewe Christian discourse) dans lequel le Diable est considéré comme le chef de tous les dieux et esprits païens (the Lord of 'pagan' gods and ghosts), ces derniers étant intégrés dans la diabologie chrétienne. De même, la sorcellerie (witchcraft ou adze en ewé) et les pratiques magico-traditionnelles (dzo), ainsi que le culte des ancêtres (adefiewo) sont considérés et combattus en tant qu'activités sataniques. Selon une conception piétiste du monde, le lien entre les mots et le monde (link between World and Word) s'exprime à travers la Bible et les enjeux de sa traduction. La lecture de la Bible ne sert pas à nourrir des analyses, mais à affermir la foi. Certains passages contiennent des vérités divines qui peuvent être reliées à la vie quotidienne et les mots (word), en particulier ceux de la Bible, peuvent provoquer une action 'dans le monde' (world). Pour B.M., cette conception pragmatique contredit la conceptualisation moderne du langage comme système de signes arbitraires dans lequel les mots sont séparés des choses qu'ils désignent (things) et, en cela, se révèle très proche de la conception Ewe du langage qui attribue aux mots un pouvoir d'influence sur les choses (p. 38). La Bible n'est pas uniquement uti-

(2) Les Peki appartiennent au groupe Ewé. Leur zone de peuplement s'étend du sud-est du Ghana au sud du Togo.

(3) L'auteur fait ici allusion à Jean \& John Comaroff, Of Revelation and Revolution: Christianity, Colonialism, and Consciousness in South Africa, vol. 1., Chicago, University of Chicago Press, 1991. 
lisée pour comprendre les choses qui ne sont pas claires, mais elle transcende (aussi) la vie quotidienne en général.

Les premiers chapitres de l'ouvrage, centrés sur l'analyse des représentations et des malentendus qui résultent de la "vernacularisation » des notions du vocabulaire chrétien, débouchent sur une analyse des conflits et dissonances qui émergent bientôt de la collaboration entre missionnaires et pasteurs africains et qui vont donner naissance à plusieurs Églises dissidentes, dont la Lord's (Pentecostal) Church ou Agbelengor en 1961, qui sera longuement analysée. Les deux autres scissions de l'EPC ont également donné naissance à l'Apostle's Revelation Society en 1949 et à l'EPC 'of Ghana' en 1991. Cet aspect de l'histoire des Églises africaines permet d'aborder la problématique de l'appropriation du pentecôtisme africain de l'intérieur des Églises et à partir des débats qui les travaillent.

\section{Des Églises missionnaires « indigènes »}

Si l'on doit aux missionnaires les premiers écrits sur l'histoire des Églises et missions en Afrique, il est aussi une production littéraire des pasteurs et dirigeants religieux africains qui, dans le cadre de thèses de philosophie religieuse ou de théologie, rassemble des données de type historiographique et des analyses sociologiques et anthropologiques qui sont de bonnes ressources pour les spécialistes de la religiosité africaine. L'ouvrage de E.K.L. constitue en fait une partie de la thèse soutenue par l'auteur à l'université d'Édimbourgh en 1995 (4). L'avant-propos de Paul Gifford, le spécialiste connu du pentecôtisme au Ghana, souligne l'ambition de l'auteur de pallier les manques d'une historiographie de la religiosité africaine jusque-là consacrée aux seules Églises et missions dites «classiques » en centrant son analyse sur les voies de l'appropriation africaine du christianisme au sein du pentecôtisme ghanéen. La majeure partie de l'ouvrage porte sur les itinéraires religieux de deux personnages considérés comme les "pionniers du pentecôtisme ghanéen" (5) et, à travers eux, sur la formation de trois des quatre plus importantes dénominations pentecôtistes du Ghana, l'Apostolic Church, la Christ Apostolic Church et la Church of Pentecost. L'accent est mis sur les interactions qui ont amené les deux dernières dénominations à s'émanciper de la double tutelle coloniale et missionnaire de la première et sur l'impulsion qui a fait de la Church of Pentecost la plus importante dénomination pentecôtiste du Ghana (6).

La perspective d'E.K.L., ghanéen et lui-même de culture pentecôtiste, se nourrit de littérature missionnaire et théologique, habilement combinée avec les

(4) The development of Ghanaian Pentecostalism: a Study in the Appropriation of the Christian gospel in the 20 th century Ghana setting with Special Reference to the Christ Apostolic Church, the Church of Pentecost Etc., Ph. D. Diss., Centre for the Study of Christianity in non-western World, University of Edinburg, 1995.

(5) L'expression est de Robert W. Wyllie, « Pioneers of Ghanaian Pentecostalism: Peter Anim and James Mc Kweon », Journal of Religion in Africa, VI, n 2, 1974, pp. 109-122.

(6) Et la deuxième Église chrétienne après l'Église catholique, cf. Gerrie TER HAAR, « Standing up for Jesus: A survey of new developments in Christianity in Ghana », Exchange 23 : 3, 1994, p. 224. 
approches historiques des religions africaines (Debrunner, Turner, Peel), et d'anthropologie religieuse (Gifford, ter Haar), comme en témoigne la composition bibliographique de l'ouvrage. L'auteur met à profit les ressources de l'anthropologie anglo-saxonne sur ces sujets ainsi que les écrits de pasteurs africains souvent inaccessibles hors d'Afrique. Ce maniement des sources tient d'une part à la culture propre de l'auteur (qui est à prendre en compte autant que pourrait l'être la culture religieuse d'un anthropologue) et à la nature de l'objet de recherche d'autre part. Celui-ci imposait le recueil de données historiques et statistiques dont les "chefs religieux" se font aujourd'hui les dépositaires, ainsi que le recours à la littérature propre aux Églises, leur documentation interne, correspondance, textes et conventions, rapports spécifiques d'activités missionnaires, etc. L'usage différencié de ces sources permet à l'auteur d'adopter des postures tour à tour historique, théologique et sociologique. Mais ce qui domine c'est le choix délibéré de comprendre de l'intérieur l'expérience humaine et religieuse de ces «fondateurs d'Églises » (7), pour rendre compte de la cohérence des mécanismes et des tensions qui sont au fondement de la constitution de "mouvements" émergents en "Églises" fermement établies. De même qu'Harvey Cox voyait dans ses divisions internes la dynamique du mouvement pentecôtiste (8), l'on peut dire que l'histoire des jeunes Églises ghanéennes se construit sur les dissidences successives qui jalonnent l'expansion du mouvement. La première partie de l'ouvrage est centrée sur deux figures principales : le ghanéen Peter Anim et le missionnaire écossais James McKeown, tous deux d'éducation presbytérienne (presbyterian upbringing) et respectivement fondateurs de la Christ Apostolic Church (1939) et de la Church of Pentecost (1953). À travers ces portraits biographiques se tisse une histoire complexe qui apporte une intelligibilité globale du contexte transnational d'émergence du pentecôtisme en Afrique de l'Ouest et des échanges entre ses deux foyers principaux : le Nigeria et le Ghana.

Dans la filiation historique du pentecôtisme par rapport à la culture méthodiste, l'auteur présente le prophète libérien W.W. Harris comme un précurseur du mouvement pentecôtiste (9) au Ghana, les premières Églises étant elles-mêmes issues du mouvement amorcé par Harris, comme l'illustre l'Église des douze apôtres (Twelve Apostles Church). Mais dans la typologie de l'auteur, c'est la seconde génération d'Églises qui appartient au mouvement pentecôtiste proprement dit. Cette génération est marquée par Peter Anim et son organisation, la Faith Tabernacle Church (1922) dont émergeront trois des quatre têtes de file du pentecôtisme ghanéen : la Christ Apostolic Church (Peter Anim), l'Apostolic Church (Bradford) et la Church of Pentecost (James McKeown). Très tôt, le Nigeria et le Ghana se trouvent engagés dans un échange indirect dans lequel les médiations britannique et américaine jouent un rôle déterminant. Le ghanéen Peter Anim, par le biais de son affiliation avec le mouvement américain Faith Tabernacle entretient une correspondance régulière avec le dirigeant de la Faith Tabernacle Church du Nigeria qui a des assem-

(7) Une manière d'aborder l'histoire des Églises africaines que l'on trouve déjà dans l'ouvrage du nigérian Joseph A. OмоYаJowo, Makers of the Church in Nigeria (1842-1947), Lagos, Nigeria, CSS Bookshops Ltd., 1995. La Church of Pentecost elle-même, restée longtemps en dehors des travaux la concernant, prend aujourd'hui ce genre d'initiative, comme en témoigne la publication d'un texte intitulé, The Gallant Soldiers of The Church of Pentecost. History of the Fathers of Old Whose Relentless Efforts Gave Birth to the Church, par le Rev. Oppong AsARE-DuAH, Accra, Pentecost Press, 2002.

(8) Harvey Cox, Retour de Dieu. Voyage en pays pentecôtiste, Paris, Desclée de Brouwer, 1994.

(9) Inspiré en cela par les textes de David A. SHAnK, notamment, Prophet Harris, The 'Black Elijah' of West Africa, Leyde-New York-Cologne, E.J. Brill, 1994. 
blées à Lagos, Abeokuta et Ibadan. C'est à l'initiative de ce dernier qu'un contact est établi avec l'Église apostolique britannique (Bradford) et que des missionnaires sont envoyés au Nigeria afin d'étudier la possibilité d'une affiliation des pasteurs nigérians à l'Apostolic Church. Le pasteur ghanéen met alors à profit la venue des missionnaires britanniques, de passage à Accra avant de rejoindre le Nigeria, pour négocier sa propre affiliation à l'Église de Bradford. C'est cette démarche et l'affiliation obtenue en 1935 qui seront la clé de voûte du développement ultérieur du mouvement pentecôtiste ghanéen, qui se traduira par l'envoi du missionnaire James McKeown venu rejoindre le mouvement amorcé par Peter Anim. Le récit détaillé de l'auteur donne toute la mesure des échanges entre le Nigeria et le Ghana, et de la similitude du processus de développement du jeune mouvement pentecôtiste. Dans les deux pays, quasi simultanément et par des effets de miroir, les affiliations à des mouvements américains, comme le Faith Tabernacle, devancent les initiatives des missionnaires britanniques, lesquels se trouvent confrontés sur le terrain aux effets d'un fondamentalisme américain réinvesti avec force par les pasteurs africains, en particulier à propos de la «no medication doctrine ». Le scénario du malentendu des médiations américaine et britannique auprès des pasteurs africains se répète à plusieurs reprises et simultanément au Ghana et au Nigeria, comme 1'a montré John Peel (10), pour la situation nigériane.

La question épineuse du statut des «pasteurs noirs » formés et nommés par les missionnaires (blancs) au sein d’Églises affiliées à des Églises européennes, illustre d'autres malentendus internes aux Églises. E.K.L. aborde cette question, mettant en avant l'attitude paradoxale de l'Apostolic Church de Bradford à l'égard des pasteurs formés par son missionnaire James McKeown. La posture de l'Église est illustrée par sa propre Constitution citée par l'auteur: "The Constitution also stipulated that an African could be an apostle to blacks but not an apostle of the whole church. The Executive of Bradford Missionary Council is said to have previously passed a motion that a white missionary who was not an Apostle could not work under an African Apostle » (p. 213). De ce point de vue, James McKeown aurait affiché dès les débuts de son œuvre missionnaire au Ghana, y compris dans la période de son appartenance à l’Église Apostolique britannique, une volonté ferme de promouvoir des pasteurs africains à la direction de ses assemblées. Christine Leonard, dans son livre $A$ Giant in Ghana, fait allusion à la volonté du missionnaire de bâtir «une Église en héritage» qui demeurerait (après lui) la propriété de ses collaborateurs africains c'est-à-dire une Église autonome dans sa gestion et ses ressources : "From the beginning, James [McKeown] wanted the Church in Ghana to be indigenous, with African culture, ministry and finance (11)». On mesure ici la contradiction d'une Église africaine qui se pense "indigène", tout en assumant le leadership prolongé du missionnaire-fondateur (blanc) au-delà même de l'indépendance du pays (1962-1982). Mais la nature des relations du missionnaire avec sa hiérarchie (son refus de la Constitution de 1935, le statut des "pasteurs noirs", le rôle des femmes dans l'Église, et autres désac-

(10) John D. Y. PeEl, Aladura: A religious Movement Among the Yoruba, Oxford, Oxford University Press, 1968.

(11) Christine Leonard, A Giant in Ghana. 3,000 Churches in 50 Years. The Story of James McKeown and The Church of Pentecost, Chichester, New Wine Press, 1989, p. 69, cf. également pp. $70-71$. 
cords) sont, pour E.K.L., de portée mineure. Pour cet auteur, c'est le jeu de l'association (externe) avec les groupes américains qui provoque la division (interne).

\section{Camps de prière et charisme institutionnel}

Si le propre du mouvement pentecôtiste est de s'être construit historiquement sur ses dissidences successives, on comprend pourquoi ces Églises sont ellesmêmes travaillées par les risques de scission potentielle qu'elles portent en germe. En marge de son succès, la Church of Pentecost rencontre un problème particulier : le statut des prophètes-guérisseurs indépendants et du Ministère de délivrance au sein de l'Église. Dès les premiers échanges, de graves divergences apparaissent également sur la question du recours à la médecine (" non use of medicine for healing », p.110). Bien avant l'arrivée de McKeown au Ghana, le mouvement fondamentaliste américain avait posé les bases d'un extrémisme radical concernant la guérison divine et le non recours à la médecine. C'est sur la base de ces divergences que le Ghanéen Peter Anim, fermement attaché à ces enseignements, se sépare de McKeown pour créer sa propre Église, la Christ Apostolic Church en 1939, surnommée l'Église-sans-médicament («no medication church»), où la maladie est entièrement prise en charge par les «prophètes-guérisseurs" (healing prophets), une appellation trompeuse puisque ces agents se veulent aux antipodes des devinsguérisseurs et de leurs fétiches.

L'auteur consacre, à partir d'études de cas (pp. 367-416), un chapitre complet au débat sur la place des Centres et Camps de prières, de guérison et de délivrance («Pentecostalist Prayer Camps and Prayer Centres ») qui travaille encore actuellement cette Église. Là encore, c'est à travers l'approche biographique que l'auteur choisit d'aborder le phénomène des camps de prière qui firent leur apparition au sein de la Church of Pentecost à l'initiative de Gilbert A. Lawson au début des années cinquante sous la forme de "healing posts". La constitution même des camps dont les activités gravitent autour d'une personne-clé, le "prophète-guérisseur » détenteur de tous les charismes et surtout du pouvoir de guérison, remet inévitablement en question la prétention de l'Église à contenir les tentations autonomistes de ces leaders "charismatiques". Le caractère partiellement autonome de ces camps porte en lui-même un risque de scission qui ne tarde pas à se confirmer sous l'impulsion de plusieurs leaders de la Church of Pentecost, comme l'initiateur luimême, G. A. Lawson, qui amorça le mouvement en faisant d'un camp de prières une Église autonome, la Divine Healer's Church (1958) ou plus récemment, Paul Owusu Tabiri (12) qui quitta la Church of Pentecost pour créer le Bethel Prayer Camp (1995) devenu The Bethel Prayer Ministry International, aujourd'hui largement implanté dans et hors du Ghana.

Ce débat concernant les camps de guérison rejoint les analyses de B.M. à propos des « groupes de prière » (prayer groups) de l'Evangelical Presbyterian Church qui ont donné lieu également à plusieurs scissions (pp. 112-140). Dans une partie

(12) Ses postures sur la délivrance l'opposèrent à la Church of Pentecost et furent l'argument principal de la dissidence de 1995. 
intitulée « Three churches out of one », l'auteur retrace l'histoire de Samuel Yao Amedzro, originaire de Blengo (Peki-Ewe) et fondateur de l'Église Agbelengor qui, après un épisode de maladie et une guérison en 1958 dans un groupe de prières de Tekrom, s'associe avec plusieurs membres, eux-mêmes issus de l'EPC et de l'Église méthodiste pour créer le Tekrom prayer group. Le groupe de prière contraste avec la sobriété (sober) de l'EPC et de l'Église méthodiste sur plusieurs points; usage de tambours (drummed), frappement des mains (clapped hands), danse (danced), instauration de la dîme (tithes), " parler en langues » (speaking in tongues), prières de guérison (prayer healing). Les personnes malades n'étant pas autorisées à se rendre à l'hôpital ou à prendre des médicaments occidentaux, elles sont prises en charge par la prière. Dans les procédés de guérison, de prévention et de protection, on relève l'usage associé de la Florida Water, sorte d'eau de Cologne [en français dans le texte] qui permet de chasser les esprits, d'huile (oil), qui protège ceux qui en portent des esprits et sorciers, de bougies (candles) afin de détourner les esprits, d'eau bénite (Holy water) pour son pouvoir de guérison, d'encens (incense) et d'un collier orné d'une grande croix (necklace with a big cross) afin de repousser les démons. L'usage de ces recours magiques révèle que le groupe de prière prend les démons très au sérieux (p. 113). Les objets « magiques » énoncés ici rappellent ceux utilisés par l’Église du Christianisme Céleste et évoquent une possible influence des Églises Aladura du Nigeria dans le Ghana des années soixante. Sur le plan liturgique également, le groupe marque sa différence par rapport à son Église-mère : pas de prêches (sermon texts) dans ces assemblées, mais des prophètes et prophétesses, reconnus comme tels, qui racontent à l'assemblée leurs rêves et visions qu'ils considèrent comme inspirés de la puissance du Saint-Esprit et qu'ils interprètent eux-mêmes. La place accordée aux rêves et visions des prophètes rappelle une fois de plus les pratiques de l'Église du Christianisme Céleste. Ces pratiques sont ponctuellement observables dans l'Église de Pentecôte mais ne sont pas réservées aux prophètes (et il n'y a pas de « visionnaires » dans cette Église) et sont plus souvent le fait de pasteurs ou plus rarement, de témoignages de fidèles. La plasticité de ces pratiques contribue à rendre floues les catégories de la typologie locale, confortée par les discours scientifiques opposant les Églises «spirituelles » et « charismatiques ».

L'EPC qui n'apprécie pas le groupe de prière Agbelengor, joint ses forces à l'Église méthodiste pour lutter contre leur ennemi commun (common enemy). L'administration de l'EPC accuse le groupe de prière de violer les règles de l'Église et d'ignorer le monopole de l'Église en matière d'organisation de la vie chrétienne par le recours à des formes liturgiques inhabituelles (unusual methods of prayer). Peu de temps après la scission de 1961, le groupe de prières prend le nom de Lord's Church surnommée Agbelengor (en pays Peki) et abolit l'usage de la Florida Water, des bougies et croix, prétextant qu'il n'y a pas de nécessité de médiation par objets pour la bénédiction. Le Centre de prière (healing centre) qui pratique la délivrance, est fréquenté aussi bien par des membres de Agbelengor que par les membres d'autres Églises. B.M. utilise tour à tour les termes 'healing centre', 'healing station' (E.K.L., celui de 'healing post'). La médecine traditionnelle, pratiquée par les herbalists et jujumen, reste cependant le premier recours face à la maladie, la conversion ne venant que plus tard dans la quête de guérison. On comprend, à la description de l'auteur, que le Tekrom prayer group offrait déjà une structure d'accueil des malades résidant parfois plusieurs mois. Plus tard, les 
assemblées d'Agbelengor aménageront des camps de malades selon le même modèle : " many Agbelengor congregations have constructed a house in the station area to lodge their sick. Some people stay there for a long period » (p. 115).

Le décès du fondateur et chef unique d'une Église et la crise du leadership qui en résulte, contribuent à exacerber ses tensions. Ce processus, que toutes les Églises «à fondateur unique » connaissent, est étroitement lié à la question de la concentration des charismes par le fondateur et à l'héritage (légitime) des charismes que ses successeurs peuvent revendiquer (13). Le décès du fondateur est alors l'occasion d'une forme de restructuration de l'Église, qui tente de s'affirmer en tant qu'institution religieuse. Une dizaine d'années après l'indépendance du groupe de prières, le décès du fondateur John Sam Amedrzo, au début des années soixantedix, provoque une crise de doute sur l'efficacité des méthodes de guérison de l'Église («period of severe doubt about the efficacy of the Church's healing methods » p. 116). Peu de temps après, l'interdiction du recours à la consultation médicale et aux traitements médicaux fut abolie. Ces divergences vis-à-vis de l'usage de la médecine occidentale rappelle très fortement les positions de Peter Anim et le débat qui l'opposa au missionnaire James McKeown dans les années quarante. Cette divergence, nous l'avons vu, avait déjà abouti à la dissidence des groupes de Peter Anim et McKeown. K.L. ajoute cependant que l'Église de Peter Anim, restée longtemps sur ces positions «radicales », y trouva les limites de son expansion et dût se contraindre à adopter un discours plus ouvert concernant le recours médical en permettant à ses fidèles de consulter "éventuellement» un médecin en cas de maladie grave. Dans le cas d'Agbelengor, certains membres ont interprété ce changement comme le signe que l'immense pouvoir spirituel dont était capable Amedzro à ses débuts avait cessé. Pour la Christ Apostolic Church, en revanche, la levée de l'interdit a permis à cette Église d'attirer un plus grand nombre de convertis, même si, le « retard» accusé l'a maintenu toujours loin derrière la Church of Pentecost, largement plus développée : la divergence des leaders concernant le recours à la médecine a nourri un rapport de concurrence qui s'est traduit presque immédiatement par le transfert de nombreux fidèles de la Christ Apostolic Church vers le groupe de James McKeown. Notons que ce changement de position intervient durant la même décennie dans la Christ Apostolic Church et Agbelengor.

\section{Une nouvelle étape de la « pentecôtisation»}

Après les années 1980, Agbelengor, qui avait commencé par être un mouvement laïque (lay movement) commença à employer des jeunes pasteurs formés dans les collèges bibliques d'Accra (Pentecostal Bible colleges). C'est en 1985 que l'Église abandonne le nom de Agbelengor et prend le nom complet de Lord's

(13) Là encore, la comparaison avec l'histoire des Églises africaines comme l'Église du Christianisme Céleste, qu'aucun des auteurs n'évoque, est frappante, cf. les analyses d'André MARY, " Pilgrimage to Imeko (Nigeria): An African Church in the time of the 'global village' », International Journal of Urban and Regional Research, vol. 26.1, mars 2002, pp. 106-120. 
Pentecostal Church, pour signifier que l'Église n'est pas une association "ethnique" mais nationale. L'ajout du terme " Pentecostal » constitue un changement théologique décisif; il implique le renoncement à l'usage d'objets médiateurs dans le contact avec Dieu ou dans les processus de guérison et de protection, comme c'est le cas dans les Églises Indépendantes et spirituelles africaines (Aladura). L'eau, l'huile, les bougies, croix et encens qu'Agbelengor utilisait depuis les années 1960, sont évacués en 1980. La confiance en ces objets est transférée au texte biblique cité aussi bien pour évoquer le Saint-Esprit que pour chasser les démons. Dans la lignée du pentecôtisme dominant dans les collèges bibliques de formation des jeunes pasteurs, l'usage de croix est dénoncée comme "idolâtre" et "occulte" (« 'idolatrous' and 'occultistic'»). Pour les chrétiens (évangéliques) ce n'est pas dans la puissance des objets mais dans celle de la Bible (Biblical Word) que se trouve le pouvoir de l'Esprit, qui travaille ('the Holy Spirit worked'). Selon B.M., au Ghana, ce point va s'imposer comme une ligne de séparation entre les Églises pentecôtistes et les Églises 'spirituelles', les premières accusant les secondes de placer leur confiance dans la magie des objets et d'être des groupes occultes ('occult groups') succombant aux machinations du Diable. Ainsi, au cours du temps, Agbelengor opéra un profond changement en passant du statut d'Église 'spirituelle' indigène locale à celui d'Église pentecôtiste, nationale d'abord, puis internationale. Depuis 1992, elle est devenue membre du Conseil des Églises pentecôtistes du Ghana (Christian Council of Ghana Pentecostal Churches (14)).

Selon les interprétations de B.M., le groupe de prière initial fut fondé par des fidèles de l'EPC qui, en crise personnelle et en l'absence des secours de leur Église ou de la religion traditionnelle Ewe, se sont constitués en corps indépendant. D'autres membres des groupes de prière, surtout des femmes, s'y joignent pour les mêmes raisons. Le groupe de prière peut ainsi être lu comme une critique de la conception de la vie chrétienne de l'EPC (EPC's understanding of Christian life) et du modèle liturgique introduit par les missionnaires piétistes allemands. Le commentaire des Écritures par les pasteurs traditionnels formés à École Biblique (trained pastors), laisse peu de place au contact avec l'Esprit Saint, à la puissance des rêves et des visions. Avec Agbelengor, par contraste, des prophètes (et prophétesses), qui se voient eux-mêmes comme les réceptacles de la puissance de l'Esprit, révélant leurs rêves et visions (et les interprétant eux-mêmes), se mettent à occuper une place centrale. Les données apportées par B.M. sur les prophètes d'Agbelengor renvoient à d'autres analyses concernant la place des prophètes dans les Églises africaines et les ambiguïtés relatives à leur statut au sein de ces Églises (15). De ce point de vue, la Church of Pentecost du Ghana n'échappe pas, une fois de plus, au débat: le nombre restreint de "Prophètes» dans cette Eglise, et leur statut «à part » témoignent d'un sentiment frileux à l'égard de ces fonctions au sein de l'institution religieuse. Il n'y a actuellement que peu de prophètes dans cette Église, essentiellement au Ghana. Les autres prophètes (ou prophétesses) que l'on peut rencontrer dans l'Église, comme en Côte-d'Ivoire par exemple, sont des prophètes dirigeants des camps de prière et de guérison « associés » à l'Église, c'est-à-dire qui travaillent en collaboration avec les pasteurs de l'Église de Pentecôte et accueil-

(14) Auquel appartiennent également les grandes Églises nationales comme la Church of Pentecost, les Assemblies of God, l'Apostolic Church et l'Église Elim.

(15) Voir le dossier de Social Compass, «Prophètes, visionnaires et guérisseurs de l'Afrique subsaharienne contemporaine $»$, vol. $48, \mathrm{n}^{\circ} 3,2001$. 
lent un grand nombre de fidèles en provenance de cette Église, et d'autres, sans y être intégrés en tant que tels. C'est également le cas des "croyants-guérisseurs » des Assemblées de Dieu du Burkina Faso qui, bien que rattachés, ou associés, à leur Église (16), reçoivent les fidèles de toutes confessions et ne sont pas considérés comme des membres du corps pastoral de l'Église.

Dans une perspective plus ethnographique et sociologique, B.M. consacre une partie de son ouvrage à une série d'études de cas de conversion (pp. 180-212), la conversion étant le plus souvent, dans ces Églises, liée à une demande de guérison. Le processus de guérison met en cause les esprits païens éwé ( $a d z o$ ), les « démons de la famille», ou Akan (Akan spirits) et "Mamie Wata» et la conversion implique alors, par le rejet de la société traditionnelle, la rupture des convertis avec leur famille, forme sociale traditionnelle, garante des pratiques communautaires, mais aussi foyer de la sorcellerie. La délivrance, synonyme de « rupture totale avec le passé » (17), comprend deux dimensions : la première correspond à la délivrance du passé personnel (immediate past), la seconde à une libération de la "malédiction ancestrale » (ancestral past). Ces schèmes interprétatifs sont au centre des analyses de l'auteur sur les formes de l'appropriation du discours pentecôtiste qui intègre, tout en les niant, les représentations traditionnelles africaines qu'il reformule. Comme le résume assez bien l'auteur, dans une autre de ses contributions : « le Démon œuvre à travers les liens du sang; le Dieu chrétien les rompt» (18). La perception pentecôtiste du monde comporte en elle-même les éléments d'une reconstruction identitaire qui amène l'auteur à s'interroger sur le rôle des missionnaires dans la construction des identités ethniques. Sur cette question, l'auteur consacre un texte beaucoup plus récent consacré à la politique culturelle des missionnaires et à leur contribution dans l'émergence d'une "nation Ewé » (Ewe nationhood) (19), à la manière dont J. D. Y. Peel montre le rôle des missionnaires dans l'invention chrétienne de l'identité Yoruba (20).

L'approche très ethnographique de B.M. implique un tel travail de compréhension « du dedans » concernant les mécanismes de scission, de formation de nouveaux groupes et des tensions qui les travaillent, que l'on ne peut que regretter qu'une telle approche ait pour conséquence (et peut-être pour condition ?) de focaliser l'attention de l'auteur sur un seul type d'Eglise. Cette étude, en effet, ne fait pas allusion, ou si peu, aux autres Églises ghanéennes ou implantées au Ghana. Les points de comparaison d'Agbelengor avec d'autres Églises ghanéennes ou africaines sont pourtant nombreux, aussi bien sur le plan de la description rituelle que sur l'organisation de la vie chrétienne et des débats qui surgissent dans la rencontre avec les autres mouvements pentecôtistes et néo-pentecôtistes émanant notamment

(16) Cf. les analyses de Pierre-Joseph Laurent, notamment « The Faith-healers of the Assemblies of God in Burkina Faso: Taking Responsibility for Diseases Related to "Living Together" » in Social Compass, vol. 48, 2001, pp. 333-351.

(17) L'expression est de l'auteur, cf. Birgit MEyer, "'Make a complete break with the past.' Memory and post-colonial modernity in ghanaian pentecostalist discourse ", Journal of Religion in Africa, XXVI, 4, 1997, pp. 1-34.

(18) Cf. Birgit Meyer, «Les Églises Pentecôtistes Africaines, Satan et la dissociation de 'la tradition' ", Anthropologie et Sociétés, vol. 22, n 1, 1998, pp. 63-83.

(19) Birgit Meyer, « Christianity and the Ewe Nation : German Pietist Missionaries, Ewe Converts and the Politic of Culture », Journal of religion in Africa, 32.2, 2002, pp. 167-199.

(20) John D. Y. Peel, Religious Encounter and the Making of the Yoruba, Bloomington (In.), Indiana University Press, 2000. 
du Nigeria. Ce pays est, en effet, un producteur de « figures de pasteurs », d'une abondante littérature évangélique, et, depuis peu, d'un cinéma populaire dont l'influence au Ghana a fait l'objet d'une contribution plus récente de l'auteur (21).

L'ouvrage de E.K.L., fidèle à son approche historiographique, a le mérite d'aborder la dernière étape du mouvement pentecôtiste ghanéen, celle du néopentecôtisme initié par quelques grandes figures (22) comme N. Duncan-Williams, converti dans l'Église de Pentecôte et fondateur du mouvement Christian Action Faith Ministries dès 1979 ou Mensa A. Otabil, fondateur de l'International Central Gospel Church (1984) et Charles Agyin Asare, également membre de l'Église de Pentecôte et fondateur de la Word Miracle Church International (1995). Ces derniers sont eux-mêmes profondément influencés par la littérature évangélique d'auteurs comme le nigérian Benson Idahosa, le coréen Yonggi Cho, l'américain T. L. Osborn ou l'allemand Reinhard Bonnke. Les concepts de l'Évangile de la Prospérité (Prosperity Gospel) ou Abundant Life Gospel, dénonçant les démons africains de la pauvreté, sont largement diffusés et véhiculés par les croisades panafricaines. L'exposé détaillé des influences réciproques de ces auteurs-leaders rend toute la complexité du paysage religieux pentecôtiste dans et au dehors des foyers ghanéens et nigérians, dans le reste de l'Afrique de l'Ouest où la littérature et les idées précèdent l'implantation des Églises en amorçant de nouvelles tendances, de nouvelles scissions et de nouveaux mouvements par un effet d'aller-retour entre ces pays, et par extension, entre les États-Unis, l'Europe et l'Afrique. Il rassemble une somme de documentation et d'informations qui permettent de mieux comprendre les processus dynamiques qui sont au fondement du mouvement pentecôtiste dans sa dimension panafricaine et transnationale. La présentation centrée sur les portraits de leaders permet de mettre en lumière la biographie historique qui sous-tend l'expansion des pentecôtismes africains. L'approche retenue par l'auteur, dans son souci de reconstituer au plus près la pensée et le point de vue propre de ces « acteurs religieux », peut néanmoins susciter quelques réserves méthodologiques concernant le degré de fiabilité des récits et entretiens recueillis auprès des dirigeants actuels de ces Églises compte tenu du temps écoulé et de la part de mythologie que comportent toujours ces types de récits. Les deux ouvrages constituent, par la somme et la nature des données rassemblées, une ressource considérable dans la compréhension des catégories de la pensée à la fois chrétienne et africaine. Mais surtout, ils nous éclairent sur le rôle de diffusion de certains pays dans la dynamique transnationale des pentecôtismes africains, comme peuvent l’être aujourd'hui des pays comme la Corée ou le Brésil et dont les Églises pentecôtistes s'implantent depuis peu en Afrique.

\author{
Sandra FANCELLO \\ EHESS - Marseille
}

(21) Birgit Meyer, « Prières, fusils et meurtre rituel. Le cinéma populaire et ses nouvelles figures du pouvoir et du succès au Ghana », Politique Africaine, Les figures de la réussite et imaginaires politiques, $n^{\circ} 82$, juin 2001, pp. 45-62.

(22) Dont les portraits sont inspirés de l'article de Paul GIFForD, " Ghana's Charismatic Churches », Journal of Religion in Africa, XXIV, 3, 1994, pp. 241-265. 
